\title{
Toward Precise Pulmonary Nodule Descriptors for Nodule Type Classification
}

\author{
Amal Farag, Shireen Elhabian, James Graham, Aly Farag, and Robert Falk* \\ Department of Electrical and Computer Engineering, University of Louisville \\ (*) Medical Imaging Division, Jewish Hospital, Louisville, KY, USA
}

\begin{abstract}
A framework for nodule feature-based extraction is presented to classify lung nodules in low-dose CT slices (LDCT) into four categories: juxta, well-circumscribed, vascularized and pleural-tail, based on the extracted information. The Scale Invariant Feature Transform (SIFT) and an adaptation to Daugman's Iris Recognition algorithm are used for analysis. The SIFT descriptor results are projected to lower-dimensional subspaces using PCA and LDA. Complex Gabor wavelet nodule response obtained from an adopted Daugman Iris Recognition algorithm revealed improvements from the original Daugman binary iris code. This showed that binarized nodule responses (codes) are inadequate for classification since nodules lack texture concentration as seen in the iris, while the SIFT algorithm projected using PCA showed robustness and precision in classification.
\end{abstract}

Keywords: texture descriptors, SIFT, Gabor wavelet, nodule type classification.

\section{Introduction}

Texture has been a subject of major investigation by researchers, especially dealing with imaging applications such as aerial, satellite, medical images, etc., since the texture can be defined as a function of the spatial variation in pixel gray levels [1]. In medical applications image analysis techniques have played a major role for such tasks like feature extraction, classification of normal and abnormal lung tissue, registration and segmentation. There are numerous methods within the scope of texture recognition such as parametric statistical model-based techniques, structural techniques and transform-based techniques [2]. In texture classification the main goal is to produce a map which enables classification of the input image(s) to the desired classes, object type classification in correlation to other images, etc.

There are various approaches for classification using texture, but all of the approaches fall-under linearly based or non-linearly-based approaches [2]. In this paper we investigate two approaches to extract texture information from lung nodules to automatically classify each nodule into one of four predefined categories identified in [3]. The first is an adoption of the linear-based algorithm known as the Daugman Iris Recognition Algorithm [4] and the second is the non-linear approach known as the Scale Invariant Feature Transform. These approaches and how they are used for lung nodule texture-based feature extraction will be described in details in the coming section. 
The literature is rich with information on feature extraction whether it is domain specific; human face and fingerprinting, or general; color, texture and shape. The usage of texture approaches on lung nodules of low-dose CT (LDCT) slices, to the best of authors' knowledge, is not as common. The closest related works we found to our application are the following: Hara et al. [6] used $2^{\text {nd }}$ order autocorrelation features to detect lung nodules in 3D chest images, there were no results pertaining to once the nodules were detected how to classify the nodules or if the $2^{\text {nd }}$ order autocorrelation could provide information for further analysis. In [7] local texture analysis was used for identifying and classifying lung abnormalities such as tuberculosis. The k-nearest neighbor approach was implemented to extract the feature vector from the training set and leave out the feature vector that will be classified.

A nodule is defined as a small mass or lump of irregular or rounded shape, yet this definition is ambiguous when it comes to applying it in the fields of computer vision and machine learning, for example. Samala et al. [8] defined nine feature descriptors that describe the nodule characteristics that were used in assessments by radiologists. These descriptors are: 1 . subtlety; 2. internal structure; 3. calcification; 4. sphericity; 5. margin; 6. lobulation; 7. speculation; 8. texture and 9. malignancy.

The assignment of the various nodule types can be formulated by allowing $\mathrm{I}(\mathrm{x})$ to represent a CT slice, where $\mathrm{x}=\left\{(\mathrm{x}, \mathrm{y}): 1 \leq \mathrm{x} \leq \mathrm{N}_{\mathrm{x}}, 1 \leq \mathrm{y} \leq \mathrm{N}_{\mathrm{y}}\right\}$ is a finite spatial grid supporting the slice and $x_{0}=\left(x_{0}, y_{0}\right)$ be the centroid of a detected nodule region. The main objective of our framework is to assign a nodule type $c$ to a given nodule region using texture-based descriptor $\mathcal{T}\left(\mathrm{x}_{\mathrm{o}}\right)$, where $\mathrm{c} \in\{\mathcal{J}, \mathcal{W}, \mathcal{V}, \mathcal{P}\}$ which corresponds to juxta, well-circumscribed, vascular and pleural-trail respectively. This involves two main stages: first, detecting potential nodules for the given CT slice(s); second, building the nodule descriptor for each nodule type assignment/classification. In this paper we are concerned with the second stage. It is crucial that the local features extracted from the detected nodules are robust to various deformations due to scale, noise, acquisition artifacts, contrast and local geometric distortion[1].

This paper is organized as follows: section 2 describes the feature descriptor algorithms used in the classification analysis, section 3 discusses performance evaluation; and section 4 concludes the paper.

\section{Feature Descriptors}

Distinct object matching and description is an important goal for many medical imaging and computer vision applications. The success of the object description necessitate on two main conditions: invariance and distinction. The object description methodology must be robust to accommodate for various variations in imaging conditions and in the mean time producing a distinctive characterization of the desired object.

In this paper we use two algorithm designs for feature based description, Daugman Iris Recognition algorithm and the Scale Invariant Feature Transform (SIFT), on the nodule classification of Kostis et al. [3], which groups nodules into four main categories: (1) Well-circumscribed where the nodule is located centrally in the lung without being connected to vasculature; (2) Vascularized where the nodule has significant connection(s) to the neighboring vessels while located centrally in the lung; (3) Juxtapleural where a significant portion of the nodule is connected to the pleural surface; 
and (4) Pleural tail where the nodule is near the pleural surface, connected by a thin structure. Within these four categories we recognize variations in shape between the Juxta, Pleural Tail, Vascularized and Well-Circumscribed nodules and each other.
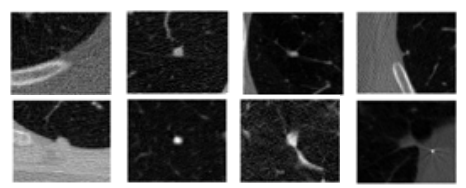

Fig. 1. Sample Nodules from Database. Column-wise: 1. Juxta; 2. Well-Circumscribed; 3. Vascualarized and 4. Pleural-tail.

This difference in shape affects the gray level texture (pattern) distribution along the surface, where the nodule centroid is densely concentrated and becomes sparser as it dissipates to the outer boundaries of the nodule. Knowing this phenomenon from nodule regions we experiment with different feature descriptors to discover whether each nodule type has prominent feature descriptions that can be extracted and used for classification. Figure 1 depicts sample nodules from our database of the four types.

\subsection{Daugman Iris Recognition Algorithm}

John Daugman in 1994 [4] developed an algorithm for use in iris recognition which is also the basis of all current iris recognition systems. In this paper we adopt a similar framework to obtain nodule codes for each nodule type to classify them into one of four categories as described by Kostis et al.[3]. The overall Daugman Iris Recognition framework adopted for Nodule Classification is depicted in Figure 2. This paper concentrates on the steps after nodule detection through feature matching. The centroid of the nodule is located for computation as if it is the pupil of the eye. The radial distribution of the entire nodule is obtained, where each concentric circle area is then demodulated to extract its phase information using quadrature 2D Gabor wavelets.

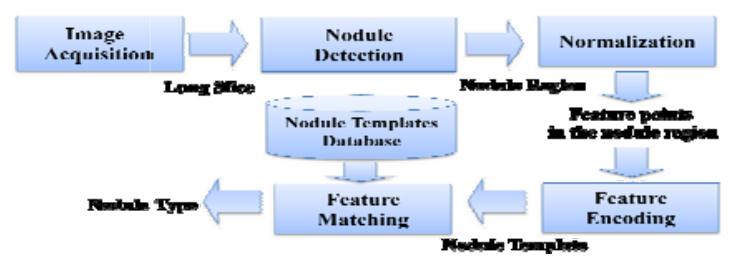

Fig. 2. Nodule Classification block-diagram adopted from Daugman's Iris Recognition framework

In Daugman's algorithm, binarization of the information was performed since the iris has concentrated texture information thus the phase binary information is enough for discrimination. In the case of the nodule we are not certain if the texture information is as concentrated as the iris, thus two techniques were implemented: (1) Daugman method with Gabor response binarization and (2) a modified Daugman method 
in which the real complex values from the Gabor response are obtained, thus bypassing binarization in nodule feature encoding step.

$$
\begin{aligned}
& h_{\{R e, I m\}} \\
& =s g n_{\{R e, I m\}} \int_{\rho} \int_{\varnothing} I(\rho, \emptyset) e^{-i \omega\left(\theta_{o}-\emptyset\right)} e^{-\left(r_{o}-\rho\right)^{2} / \alpha^{2}} e^{-\left(\theta_{o}-\emptyset\right)^{2} / \beta^{2}} \rho d \rho d \emptyset
\end{aligned}
$$

Where $\mathrm{h}_{\{\mathrm{Re}, \mathrm{Im}\}}$ can be regarded as a complex-valued bit as either 1 or 0 depending on the sign of the $2 D$ integral representing the real and imaginary parts; $I(\rho, \emptyset)$ is the raw nodule image in a dimensionless polar coordinate system that is translation and size invariant, $\alpha$ and $\beta$ are the multi-scale 2D wavelet size parameters; $\omega$ is the wavelet frequency, spanning 3 octaves in inverse proportion to $\beta ;\left(r_{0}-\rho\right)$ represents the polar coordinated of each region of the nodule for which the phasor coordinates $\mathrm{h}_{\{\mathrm{Re}, \mathrm{Im}\}}$ are computed. In the first technique the fractional Hamming Distance (HD) is computed, as conducted by Daugman. This distance is used as the dissimilarity measure between any 2 nodules, where 0 would represent a perfect match.

$$
H D=\frac{\|(\text { nodule } A \otimes \text { nodule } B) \cap \operatorname{mask} A \cap \text { mask } B \|}{\|\operatorname{mask} A \cap \operatorname{mask} B\|}
$$

Where the XOR, $\otimes$, operator detects disagreement between any corresponding pair of bits while the AND, $\cap$, operator ensures that the compared bits are both deemed to have been uncorrupted by the lung nodule surroundings. Since the second technique is unbinarized, the Hamming Distance cannot be used thus the Euclidean Distance (ED) was computed, which examines the root of square difference between any 2 nodules. Given two descriptors $\mathbf{d}_{\mathbf{i}}=\left[d_{i 1}, d_{i 2}, \ldots, d_{i N}\right]^{\mathbf{T}}$ and $\mathbf{d}_{\mathbf{j}}=\left[d_{j 1}, d_{j 2}, \ldots, d_{j N}\right]^{\mathbf{T}}$, ED can be defined as follows;

$$
E D_{i j}=\sqrt{\sum_{k=1}^{n}\left(d_{i k}-d_{j k}\right)^{2}}
$$

\subsection{Scale Invariant Feature Transform (SIFT)}

As detailed in [6], SIFT consists of four main steps: (1) Scale-space peak selection; (2) Key-point localization; (3) Orientation assignment and (4) Key-point descriptor. In the first step, potential interest points are detected using a scale-space continuous function $\mathbf{L}\left(\mathbf{x}, \sigma_{s}\right)$, it can be constructed by convolving the image $\mathbf{I}(\mathbf{x})$ with a cylindrical Gaussian kernel $\mathbf{G}\left(\mathbf{x}, \sigma_{s}\right)$ which can be viewed as a stack of 2D Gaussians one for each band. According to Lowe [5], the scale is discretized as $\sigma_{s} \in\left\{k^{s}\right\}$ where $k=2^{1 / 3}$ and $s=\left\{-1,0,1,2, \ldots, \frac{\log \left(s_{\max }\right)}{1 / 3 \log 2}\right\}$. Scale-space extrema detection searches over all scales $\sigma_{s}$ and image locations $\mathbf{x}=\{(x, y)\}$ to identify potential interest points which are invariant to scale and orientation; this can be efficiently implemented using Difference-of-Gaussians $\mathbf{D}\left(\mathbf{x}, \sigma_{s}\right)$ which takes the difference between consecutive scales, i.e. $\mathbf{D}\left(\mathbf{x}, \sigma_{s}\right)=\mathbf{L}\left(\mathbf{x}, \sigma_{s}\right)-\mathbf{L}\left(\mathbf{x}, \sigma_{s-1}\right)$, a point $\mathbf{x}$ is selected to be a candidate interest point if it is larger or smaller than its $3 \times 3$ neighborhood system defined on 
$\left\{D\left(\mathbf{x}, \sigma_{s-1}\right), D\left(\mathbf{x}, \sigma_{s}\right), D\left(\mathbf{x}, \sigma_{s+1}\right)\right\}$, where $\sigma_{s}$ is marked to be the scale of the point $\mathbf{x}$. This process leads to too many points some of which are unstable (sensitive to noise); hence removal of points with low contrast and points that are localized along edges is accomplished. In our framework, we assume that nodules have been already detected which correspond to interest/key points in Lowe's algorithm, hence this step can be bypassed. In order to obtain a nodule SIFT descriptor which is invariant to orientation, a consistent orientation should be assigned to the detected nodule which is represented by its centroid $\mathbf{x}_{\mathbf{0}}$. This orientation is based on the gradient of the nodule's local image patch. Considering a small window surrounding $\mathbf{x}_{\mathbf{o}}$, the gradient magnitude and orientation can be computed using finite differences. Local image patch orientation is then weighted by the corresponding magnitude and Gaussian window. Eventually the orientation is selected to be the peak of the weighted orientation histogram. Building a nodule SIFT descriptor is similar to orientation assignment, for example a 16x16 image window surrounding the nodule centroid point $\mathbf{x}_{\mathbf{o}}$ is divided into sixteen $4 \times 4$ sub-windows, then an 8-bin weighted orientation histogram is computed for each sub-window, hence, we obtain 16x8 = 128 descriptors for each nodule. Thus, each detected nodule can now be defined at location $\left(x_{0}, y_{0}\right)$, specific scale $\sigma$, explicit orientation $\theta$ and descriptor vector $\mathbf{x}_{\mathbf{o}}=\left\{x_{0}, y_{0}, \sigma, \theta, \mathbf{d}\right\}$. Thus the SIFT operator $\mathcal{S}: \mathrm{I}(\mathrm{x}) \rightarrow \mathrm{X}$ can be viewed as mapping a CT slice $\mathrm{I}(\mathrm{x})$ to the nodule space with n-nodules, $X=\left\{\mathbf{x}_{\mathbf{i}}\right\}_{\mathrm{i}=1}^{\mathrm{n}}$ detected from $\mathrm{I}(\mathrm{x})$, where $\mathbf{x}_{\mathbf{i}}=\left\{\mathrm{x}_{0}^{\mathrm{i}}, \mathrm{y}_{0}^{\mathrm{i}}, \sigma_{\mathrm{i}}, \theta_{\mathrm{i}}, \mathrm{d}_{\mathrm{i}}\right\}$. Principle component analysis (PCA) [9] and linear discriminant analysis (LDA) [10] are used to project the extracted SIFT descriptors to a low-dimensional subspace where noise is filtered out.

\section{Experimental Results}

This work is based on the Early Lung Cancer Action Program (ELCAP) public database [11], which contains 50 sets of low-dose CT lung scans taken at a single breath-hold with slice thickness $1.25 \mathrm{~mm}$. The locations of the 397 nodules are provided by the radiologists, where $39.12 \%$ are juxta-pleural nodules, $13.95 \%$ are vascularized nodules, $31.29 \%$ are well-circumscribed nodules and $15.65 \%$ are pleural-tail nodules. In this paper we created a subset database containing 294 nodules of the original 397 . The ELCAP database is of resolution $0.5 \times 0.5 \mathrm{~mm}$ [11]. Since we assume that the nodule region has been already detected, we use the groundtruth marked nodules by the radiologists to avoid sources of errors due to automated detection. Given a nodule's centroid, we extract texture descriptor information using two main techniques: Daugman coding and the SIFT descriptor. Tables 1 and 2 visualize the intermediate steps performed to generate a nodule code for the four nodule types while table 3 represents the SIFT descriptors of the nodule ensembles given in Tables 1 and 2.

Training was performed using two randomly drawn approaches; the bootstrapping resampling technique [12] and a one-time random sampling approach. The results using both methods were comparable, thus only the one time random sampling results are shown in this paper.

Classification was performed using the nearest-neighbor classifier with Euclidean distance as the similarity measure. To quantify nodule type classification performance, we measure true positives rates. A classification result is considered a true positive if a sample from class $\boldsymbol{w}_{\boldsymbol{i}}$ is classified as belonging to the same class. 
Table 1. Visualization of Daugman Recognition process for the same Juxta and Well-Circumscribed Nodules in Fig. 1

\begin{tabular}{|c|c|c|}
\hline & Juxta & Well-Circumscribed \\
\hline $\begin{array}{c}\text { Radial } \\
\text { Distribution }\end{array}$ & & 0 \\
\hline Rubber Sheet & & \\
\hline $\begin{array}{c}\text { Binarized } \\
\text { Code }\end{array}$ & & \\
\hline $\begin{array}{c}\text { Unbinarized } \\
\text { Code }\end{array}$ & & \\
\hline
\end{tabular}

Table 2. Visualization of Daugman Recognition process for the same Pleural-Tail and Vascularized Nodules in Fig. 1

\begin{tabular}{|c|c|c|c|c|c|}
\hline & \multicolumn{2}{|c|}{ Pleural-Tail } & \multicolumn{3}{c|}{ Vascularized } \\
\hline $\begin{array}{c}\text { Radial } \\
\text { Distribution }\end{array}$ & & & & & \\
\hline
\end{tabular}

Table 4 shows the classification results for nodule codes for different percentages of training data ( $\mathrm{x} \%$ is the amount of ground-truth nodules taken into consideration in the training phase). It can be inferred that binarizing Gabor wavelet complex responses makes the nodule region lose discriminatory texture which assists in nodule type classification; this is not the case for iris recognition where it is known that the iris region is rich in texture. Gabor-based descriptor provides higher discrimination for juxta and well-circumscribed nodule types when compared to vascular and pleural tail. In general $50 \%$ training data can be used to provide overall excellent classification results.

Table 5 shows the classification results for nodule SIFT descriptor for different percentages of training data. We also use the projection of SIFT descriptor on a PCAbased and LDA-based subspaces trained by the descriptors of each nodule type. It can be observed that using the raw SIFT descriptor without statistical modeling provides the worst classification performance when compared to their PCA and LDA projection, yet, it provides similar performance to complex-valued nodule codes. LDA projection provides the greatest classification results when $100 \%$ nodule training is conducted, yet, in reality the model will not be re-trained each time new nodules are extracted to then classify them again (i.e. Input everything desired, output the same as input). As training percentage decreases, PCA projection results surpass those from the raw SIFT, LDA SIFT and Daugman nodule codes, this emphasizes the ability of 
PCA as a statistical modeling approach to generalize the decision boundary between different classes (nodule types) with minimal amounts of training data. On the other hand, LDA requires larger amounts of training samples to provide a general subspace which has high discrimination power.

Table 3. Visualization of SIFT Recognition process for the same Juxta , Well-Circumscribed, Pleural-Tail and Vascularized Nodules in Fig. 1

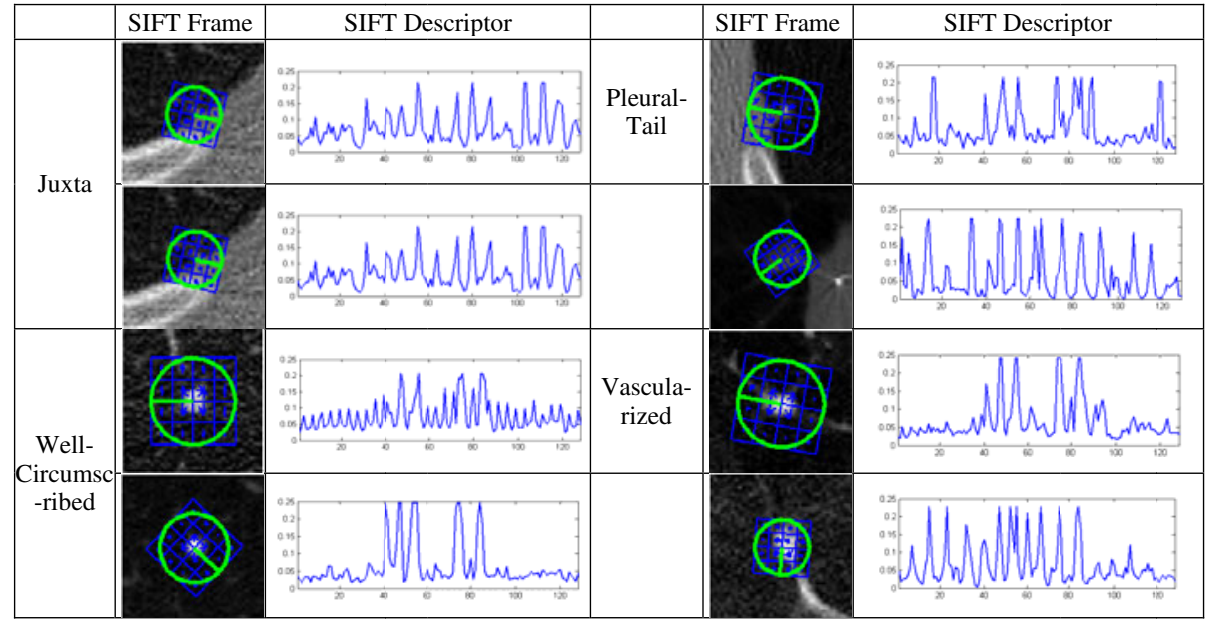

Table 4. Daugman Results for the Binarizied and Unbinarized Nodules using the Hamming and Euclidean Distances (eqs. 2 and 3)

\begin{tabular}{|c|c|c|c|c|c|c|c|c|c|c|}
\hline \multirow{2}{*}{$\begin{array}{l}\text { Training } \\
\text { Percentage }\end{array}$} & \multicolumn{5}{|c|}{ Binary Nodule Codes } & \multicolumn{5}{|c|}{ Complex-valued Nodule Codes } \\
\hline & Overall & Juxta & Well & Vascular & Pleural Tail & Overal & Juxta & Well & Vascular & Pleural Tail \\
\hline $100 \%$ & $41.84 \%$ & $50.43 \%$ & $43.48 \%$ & $19.51 \%$ & $36.96 \%$ & $56.80 \%$ & $74.78 \%$ & $60.87 \%$ & $21.95 \%$ & $34.78 \%$ \\
\hline $75 \%$ & $40.82 \%$ & $49.57 \%$ & $41.30 \%$ & $19.51 \%$ & $36.96 \%$ & $61.56 \%$ & $76.52 \%$ & $67.39 \%$ & $24.39 \%$ & $45.65 \%$ \\
\hline $50 \%$ & $40.48 \%$ & $49.57 \%$ & $43.48 \%$ & $24.39 \%$ & $26.09 \%$ & $58.50 \%$ & $73.04 \%$ & $64.13 \%$ & $21.95 \%$ & $43.48 \%$ \\
\hline $25 \%$ & $34.69 \%$ & $41.74 \%$ & $39.13 \%$ & $19.51 \%$ & $21.74 \%$ & $54.42 \%$ & $69.57 \%$ & $58.70 \%$ & $21.95 \%$ & $36.96 \%$ \\
\hline
\end{tabular}

Table 5. SIFT Results obtained from the original SIFT, and results after applying the PCA and LDA methods to the SIFT output

\begin{tabular}{|c|c|c|c|c|c|c|c|c|c|c|c|c|c|c|c|}
\hline \multirow{2}{*}{$\begin{array}{l}\text { Training } \\
\text { Percentage }\end{array}$} & \multicolumn{5}{|c|}{ Raw SIFT } & \multicolumn{5}{|c|}{ PCA SIFT } & \multicolumn{5}{|c|}{ LDA SIFT } \\
\hline & Overall & Juxta & Well & $\begin{array}{c}\text { Vascul } \\
\text { ar }\end{array}$ & $\begin{array}{c}\text { Pleural } \\
\text { Tail }\end{array}$ & Overall & Juxta & Well & Vascular & $\begin{array}{c}\text { Pleural } \\
\text { Tail }\end{array}$ & Ove & Juxta & Well & Vascular & $\begin{array}{c}\text { Pleural } \\
\text { Tail }\end{array}$ \\
\hline $100 \%$ & $58.84 \%$ & $70.43 \%$ & $72.83 \%$ & $26.83 \%$ & $30.43 \%$ & $67.69 \%$ & $77.39 \%$ & $83.70 \%$ & $31.71 \%$ & $43.48 \%$ & $88.10 \%$ & $91.30 \%$ & $90.22 \%$ & $85.37 \%$ & $78.26 \%$ \\
\hline $50 \%$ & $56.80 \%$ & 68.7 & $73.91 \%$ & $14.63 \%$ & $30.43 \%$ & $76.53 \%$ & $87.00 \%$ & $81.52 \%$ & $56.10 \%$ & $58.70 \%$ & $68.71 \%$ & $73.91 \%$ & $65.22 \%$ & $65.85 \%$ & $65.22 \%$ \\
\hline $25 \%$ & $58.23 \%$ & $64.35 \%$ & $57.61 \%$ & $12.20 \%$ & $28.26 \%$ & $78.57 \%$ & $83.48 \%$ & $82.61 \%$ & $65.85 \%$ & $69.57 \%$ & $51.36 \%$ & $60.00 \%$ & $56.52 \%$ & $39.02 \%$ & $30.43 \%$ \\
\hline
\end{tabular}

\section{Conclusion}

In this paper we investigated the effects of texture analysis using two feature descriptor algorithms; Gabor wavelet nodule responses obtained from an adopted Daugman 
Iris Recognition algorithm and the SIFT descriptor with lower-dimensional subspaces projections using PCA and LDA. The results from the descriptors were used to classify the nodules into their corresponding classes as defined by [3]. The results revealed the PCA SIFT method was more robust for lesser nodule training data. The Gabor wavelet unbinarized nodule responses provided better results than the binarized original framework of Daugman due to the lack of concentrated texture information in the lung nodules, yet these results were inferior to the overall SIFT performance. Future directions are geared toward generating a larger nodule database from other clinical data to expand our work. Further experimentations with this approach in terms of training and testing data will be conducted. We are also aiming to examine other feature descriptor approaches to compare with the results obtained in this paper.

\section{References}

1. Tuceryan, M., Jain, A.K.: Texture analysis. In: Chen, C., Pau, L., Wang, P. (eds.) Handbook of Pattern Recognition and Computer Vision, 2nd edn., pp. 207-248. World Scientific, River Edge (1998)

2. Tao, B., Dickinson, B.: Texture recognition and image retrieval using gradient indexing. Journal of Visual Communication and Image Representation 11(3), 327-342 (2000)

3. Kostis, W.J., et al.: Small pulmonary nodules: reproducibility of three-dimensional volumetric measurement and estimation of time to follow-up. Radiology 231, 446-452 (2004)

4. Daugman, J.: How iris recognition works. In: Proceedings of 2002 International Conference on Image Processing, vol. 1 (2002)

5. Lowe, D.G.: Distinctive image features from scale-invariant keypoints. International Journal of Computer Vision 60(2), 91-110.4 (2004)

6. Hara, T., Hirose, M., Zhou, X., Fujita, H., Kiryu, T.: Nodule detection in 3D chest CT images using 2nd order autocorrelation features. In: Proceedings of the 2005 IEEE Engineering in Medicine and Biology 27th Annual Conference, Shanghai, China (2005)

7. van Ginneken, B., Katsuragwa, S., Romney, B., Doi, K., Viergever, M.: Automatic Detection of Abnormalities in Chest Radiographs Using Local Texture Analysis. IEEE Transactions on Medical Imaging 21(2) (2002)

8. Samala, R., et al.: A Novel Approach to Nodule Feature Optimization on Thin Section Thoracic CT. Acad. Radiology 15, 1181-1197 (2009)

9. Shlens, J.: A Tutorial on Principal Component Analysis. Institue for Nonlinear Science. UCSD (2005)

10. Balakrishnama, S., Ganapathiraju, A.: Linear Discrimant Analysis- A brief tutorial, http://www.isip.msstate.edu/publications/reports/isip_intern al/1998/linearpublications/linear_discrim_analysis /

11. ELCAP public lung image database, http: / /www.via.cornell. edu/databases / lungdb.html

12. Polikar, R.: Ensemble Based Systems in Decision Making (2006) 